\title{
Vitamin A deficiency and child mortality in Mozambique
}

\author{
Victor M Aguayo ${ }^{1, *}$, Sonia Kahn ${ }^{2}$, Carina Ismael ${ }^{2}$ and Stephan Meershoek ${ }^{3}$ \\ 'UNICEF Regional Office for West and Central Africa, BP 29720, Dakar-Yoff, Senegal: ${ }^{2}$ Ministry of Health, Maputo, \\ Mozambique: ${ }^{3}$ Helen Keller International, Maputo, Mozambique
}

Submitted 24 November 2003: Accepted 4 August 2004

\begin{abstract}
Background: In areas where vitamin A deficiency (VAD) is prevalent, vitamin A repletion reduces child mortality by $23 \%$ on average.

Objectives: To estimate the potential child survival benefits of policies and programmes aimed at controlling VAD in Mozambique, and to make policy and programme recommendations.

Methods: The potential contribution of VAD to child mortality in Mozambique was estimated by combining the observed VAD prevalence in the under-5s $(71.2 \%)$, the measured child mortality effects of VAD (risk of death in children with VAD $=1.75$ times higher than in children without VAD) and the observed under-5 mortality rate in the country (210 per 1000 live births).

Results: In Mozambique, an estimated 2.3 million children below the age of 5 years are vitamin-A-deficient. In the absence of appropriate policy and programme action, VAD will be the attributable cause of over 30000 deaths annually in the under-5s. This represents $34.8 \%$ of all-cause mortality in this age group.

Discussion: Vitamin A supplementation (VAS) has been adopted as a short- to medium-term strategy to control VAD in children, and is integrated into routine child health services. However, the last VAS coverage survey showed that only $46 \%$ of children received a vitamin A supplement in the 6 months preceding the survey. If VAS coverage is to increase significantly in the foreseeable future, four areas appear to be of paramount importance: (1) reduce missed opportunities for VAS such as visits of sick children to child health services and community outreach activities; (2) take advantage of all potential opportunities for accelerating VAS coverage, such as additional vaccination campaigns and emergency response activities; (3) strengthen health workers' training, supervision and monitoring skills; and (4) increase community demand for VAS of children. Biannual VAS, as the primary component of an integrated strategy for VAD control in children, has the promise to be among the most cost-effective/high-impact child survival interventions in Mozambique.
\end{abstract}

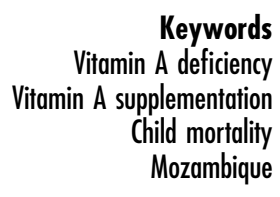

Between 1986 and 1993, eight population-based intervention trials world-wide assessed the contribution of vitamin A deficiency (VAD) to child mortality. An independent meta-analysis of these trials showed that, in areas where $\mathrm{VAD}$ is prevalent, vitamin A repletion reduces child mortality by $23 \%$ on average ${ }^{1}$. This significant reduction in child mortality is largely a result of the mortality reduction from measles, severe diarrhoea and falciparum malaria. The objectives of the present analysis are to estimate the potential child survival benefits of policies and programmes aimed at controlling VAD in Mozambique, and to make policy and programme recommendations.

\section{Methods and findings}

The most recent data on the extent of VAD in Mozambique come from the national VAD survey conducted by the Ministry of Health between December 2001 and January
2002. Blood samples were collected from a nationally representative sample of children aged 6-59 months, using a two-stage cluster sampling methodology. Serum retinol concentration was analysed using high-performance liquid chromatography. VAD was defined as a serum retinol concentration below $0.70 \mu \mathrm{moll}^{-1} ; 71.2 \%$ of children had serum retinol levels below this threshold and thus were considered vitamin-A-deficient ${ }^{2}$. Children with $\mathrm{VAD}$ are at increased risk of death; using the data from the eight population-based trials included in the meta-analysis by Beaton et al. ${ }^{1}$, it is estimated that the risk of death in 659-month-old children with VAD is 1.75 times higher than in children without $\mathrm{VAD}^{3}$. The most recent Demographic and Health Survey in Mozambique shows a nation-level child mortality rate of 201 per 1000 live births ${ }^{4}$. The potential contribution of VAD to child mortality in Mozambique was estimated by combining the observed $\mathrm{VAD}$ prevalence in under- $5 \mathrm{~s}$, the measured child mortality 
effects of VAD and the child mortality levels observed in the country. The following equation was used:

$$
\operatorname{PAR}=\operatorname{PREV}(\mathrm{RR}-1) / 1+[\operatorname{PREV}(\mathrm{RR}-1)],
$$

where PAR (population attributable risk) is the proportion of all-cause child mortality in Mozambique attributable to VAD, PREV is the observed prevalence of VAD in children (71.2\%) and RR is the increased risk of death in children with VAD relative to children without VAD (1.75). Our analysis shows that, in Mozambique, an estimated 2.3 million children under 5 years of age are vitamin-Adeficient. In the absence of appropriate policy and programme action, VAD will be the attributable cause of over 30000 deaths annually among children aged 6-59 months. This represents $34.8 \%$ of all-cause mortality in this age group (Table 1 ).

\section{Discussion}

In view of the large number of children suffering from VAD and the extraordinary contribution of VAD to child mortality in Mozambique, the Ministry of Health has adopted high-potency vitamin A supplementation (VAS) as a short- to medium-term strategy for the control of VAD in children. Mozambique's experience in VAS of children started in 1999, with the integration of VAS into National Immunisation Days (NIDs) for polio eradication. VAS coverage with NIDs attained $100 \%$ of children aged 6-59 months. NIDs were phased out in 2000 and an alternative strategy was tested. Vitamin A supplements were distributed at Mother and Child Health Days in 2000 and 2001. VAS coverage (one high-potency vitamin A supplement per year) attained $79 \%$ and $91 \%$ of $6-59$ month-old children in 2000 and 2001, respectively.

Despite these apparently encouraging results, the Government of Mozambique felt that these campaigns

Table 1 Vitamin A deficiency (VAD) and child mortality in Mozambique, 2000

\begin{tabular}{lr}
\hline Observed VAD prevalence (\%) & 71.2 \\
Births per year* & 793000 \\
Neonatal mortality rate (per 1000 live births)† & 54 \\
Infant mortality rate (per 1000 live births)† & 135 \\
Child mortality rate (per 1000 live births)† & 201 \\
Population of children 0-59 months old* & 3178000 \\
Population of children 0-59 months old & 2262736 \\
$\quad$ with VAD & \\
Neonatal deaths per annum (first month of life) & 42822 \\
Infant deaths per annum (0-11 months) & 107055 \\
Deaths of infants aged 1-11 months per annum & 64233 \\
Deaths of infants aged 6-11 months per annum & 35036 \\
Child deaths per annum (0-59 months) & 159393 \\
Deaths of children aged 12-59 months per annum & 52338 \\
Deaths of children aged 6-59 months per annum & 87374 \\
Deaths of children aged 6-59 months attributable to & 30416 \\
$\quad$ VAD per annum & \\
Percentage of deaths among children & 34.8 \\
$\quad$ aged 6-59 months attributable to VAD & \\
\hline
\end{tabular}

* Source: reference 5 [data refer to 2000].

† Source: reference 4 . put too high a demand on the limited human and financial resources of the Ministry of Health and decided to discontinue them. Beginning in March 2002, VAS was integrated into routine child health services, with a combination of fixed and mobile health posts. The goal was to ensure that all children aged 6-59 months reached by health facilities receive a high-potency vitamin A supplement every 6 months (to maximise the child survival benefits of VAS). Posters, leaflets and radio spots were developed to create demand in the population for VAS of children at every health contact.

Routine information collected in 2003 by the Ministry of Health showed that VAS coverage is $44 \%$. This means that less than $50 \%$ of children aged 6-59 months received a high-potency vitamin A supplement in the 6 months preceding the coverage survey. The main reasons for this low VAS coverage are:

1. Missed opportunities for VAS in regular health activities such as during visits of sick children to child health services and community outreach activities;

2. Missed opportunities for VAS in supplemental health activities such as additional vaccination campaigns and emergency response activities;

3. Sub-optimal training, supervision and monitoring skills of health workers in VAS for child survival; and

4. Low community demand for VAS of children.

If VAS coverage is to increase significantly in the foreseeable future, addressing these four bottlenecks appears to be of paramount importance.

Programmatically, one of the challenges is to ensure that VAS does not delay, displace or weaken the implementation of other strategies but that it drives an integrated, effective and sustained approach to VAD control that integrates:

- Improved infant and young child nutrition. Mortality data from the eight population-based field trials included in the meta-analysis by Beaton et al. ${ }^{1}$ show that mortality reduction in children aged 6-24 months made up more than $70 \%$ of the number of lives saved through improving vitamin A status in 6-59-month-old children. Optimal infant and young child nutrition is therefore crucial. Breast milk is vital in keeping infants adequately nourished with vitamin A throughout infancy and early childhood ${ }^{6}$. Evidence shows that well-designed community-based and/or facility-based behaviour change communications programmes can bring about dramatic improvements in early initiation of breast-feeding and exclusive breast-feeding rates for 6 months ${ }^{7}$. When the vitamin A content of human milk is sub-optimal, VAS of women in the early postpartum period becomes key in improving women's vitamin A status and the vitamin A content of breast milk ${ }^{8}$. Mozambique is in the process of formulating a national policy to ensure that women receive a high-potency 
vitamin A supplement as soon as possible after delivery. A number of facility-based and community-based approaches are being tested.

- Improved vitamin A dietary intake. Dietary improvement approaches need to be an integral part of a sustainable strategy to control VAD. Significant progress has been achieved in the past 10 years in the design and implementation of such approaches, particularly the new generation of projects that integrate production, nutrition education and behaviour change communication strategies 9 . In Mozambique, $\beta$-carotene-rich varieties of sweet potato are being tested and introduced. Vitamin A fortification of locally available foods can also be crucial in improving the vitamin A status of the general population and that of women of reproductive age in particular. Plans are underway to assess nation-level food consumption of potential food vehicles for vitamin A fortification, the capability for vitamin A fortification in selected food industries, and the potential for successful private-public partnerships for vitamin A fortification.

Our analysis shows that VAD control has the promise to be among the most cost-effective/high-impact child survival interventions in Mozambique; improving the vitamin A status of children could reduce child mortality in the country by an estimated 34.8\% from 1997 child mortality levels. Among the challenges that Mozambique needs to face in the coming years, VAD in children is one that can be overcome. The solutions are known, effective and affordable, and the need is more urgent than ever.

\section{Acknowledgements}

Funding source: This paper is a product of Helen Keller International. The work was carried out with a grant from the Micronutrient Initiative (MI), Ottawa, Canada, with financial assistance of the Government of Canada through the Canadian Development Agency. The opinions expressed in this paper do not necessarily reflect those of the United Nations Children's Fund, MI or the Ministry of Health in Mozambique.
Contributors: V.M.A. had the original idea for the paper, conducted the analysis and interpretation of data, and prepared the manuscript; he will act as guarantor of the paper. S.K., C.I. and S.M. contributed to the interpretation of the data and presentation of the paper.

Conflict of interest: None stated.

\section{References}

1 Beaton $\mathrm{GH}$, Martorell $\mathrm{R}$, Aronson $\mathrm{KJ}$, Edmonston $\mathrm{B}$, McCabe G, Ross AC, et al. Effectiveness of Vitamin A Supplementation in the Control of Young Child Morbidity and Mortality in Developing Countries. United Nations (UN) Administrative Committee on Coordination, Sub-committee on Nutrition State-of-the-Art Series: Nutrition Policy Discussion Paper No. 13. Geneva: UN, 1993.

2 Ismael C, Khan SG, Thompson R, Meershoek S, Van Streirteghem V. Inquérito nacional sobre a deficiência de vitamina A e prevalência de anemia e malária em crianças dos 6-59 meses e respectivas mães. Maputo: MISAU Repartição de Nutrição e Instituto Nacional de Saúde, Helen Keller International and United Nations Children's Fund, 2003.

3 Ross JS. Derivation of the Relative Risk of Child Mortality due to Vitamin A Deficiency. PROFILES Working Notes Series No. 2. Washington, DC: Academy for Educational Development, 1996.

4 Da Costa Gaspar M, Cossa HA, Ribeiro dos Santos C, Manjate RM, Shoemaker J. Moçambique, Inquérito Demográfico e de Saúde 1997. Maputo/Calverton, MD: Instituto Nacional de Estatística de Maputo/Macro International Inc., 1998.

5 United Nations Children Fund (UNICEF). The State of the World's Children 2002. New York: UNICEF, 2002.

6 Ross JS, Harvey PWJ. Contribution of breastfeeding to vitamin A nutrition of infants: a simulation model. Bulletin of the World Health Organization 2003; 81(2): 80-6.

7 World Heath Organization (WHO). Community-based Strategies for Breastfeeding Promotion and Support in Developing Countries. Geneva: WHO, 2003.

8 Roy SK, Islam A, Molla A, Akramuzzaman SM, Jahan F, Fuchs G. Impact of a single megadose of vitamin A at delivery on breastmilk of mothers and morbidity of their infants. European Journal of Clinical Nutrition 1997; 51: 302-7.

9 Ruel MT. Can Food-Based Strategies Help Reduce Vitamin A and Iron Deficiencies? A Review of Recent Evidence. Washington, DC: International Food Policy Research Institute, 2001. 